\title{
Usefulness of Magnetic Particle Imaging for Monitoring the Effect of Magnetic Targeting
}

\author{
Tomomi Kuboyabu, Akiko Ohki, Natsuo Banura, Kenya Murase* \\ Department of Medical Physics and Engineering, Division of Medical Technology and Science, \\ Faculty of Health Science, Graduate School of Medicine, Osaka University, Osaka, Japan \\ Email: "murase@sahs.med.osaka-u.ac.jp
}

Received 15 April 2016; accepted 5 June 2016; published 8 June 2016

Copyright (C) 2016 by authors and Scientific Research Publishing Inc.

This work is licensed under the Creative Commons Attribution International License (CC BY). http://creativecommons.org/licenses/by/4.0/

(c) (i) Open Access

\section{Abstract}

Purpose: Magnetic targeting refers to the attachment of therapeutic agents to magnetizable particles such as magnetic nanoparticles (MNPs) and then applying magnetic fields to concentrate them to the targeted region such as solid tumors. The purpose of this study was to investigate the usefulness of magnetic particle imaging (MPI) for monitoring the effect of magnetic targeting using tumor-bearing mice. Materials and Methods: Colon-26 cells $\left(1 \times 10^{6}\right.$ cells $)$ were implanted into the backs of eight-week-old male BALB/c mice. When the tumor volume reached approximately $100 \mathrm{~mm}^{3}$, the mice were divided into treated $(n=8)$ and untreated groups $(\mathrm{n}=8)$. The tumors in the treated group were directly injected with MNPs (Resovist ${ }^{\circledR}, 250 \mathrm{mM}$ ) and a neodymium magnet was attached to the tumor surface, whereas the magnet was not attached to the tumor in the untreated group. The mice were imaged using our MPI scanner and the average and maximum MPI values were obtained by drawing a region of interest (ROI) on the tumor, with the threshold value for extracting the contour of the tumor being taken as $40 \%$ of the maximum MPI value in the ROI. The relative tumor volume growth (RTVG) was calculated from $\left(V-V_{0}\right) / V_{0}$, where $V_{0}$ and $V$ represented the tumor volume immediately before and after the injection of MNPs, respectively. Results: The average and maximum MPI values in the treated group were significantly higher than those in the untreated group 3 days after the injection of MNPs, suggesting the effectiveness of magnetic targeting. There were no significant differences in RTVG between the two groups. Conclusion: Our preliminary results suggest that MPI is useful for monitoring the effect of magnetic targeting.

\section{Keywords}

Magnetic Particle Imaging, Magnetic Targeting, Magnetic Nanoparticles, Tumor-Bearing Mice

\footnotetext{
${ }^{*}$ Corresponding author.
}

How to cite this paper: Kuboyabu, T., Ohki, A., Banura, N. and Murase, K. (2016) Usefulness of Magnetic Particle Imaging for Monitoring the Effect of Magnetic Targeting. Open Journal of Medical Imaging, 6, 33-41. 


\section{Introduction}

Molecular transport based on magnetic nanoparticles (MNPs) has been the subject of recent strategies to enhance drug delivery and reduce drug toxicity in diverse medical fields [1] [2]. MNPs are actively being developed based on their unique properties to respond to magnetic fields including static and alternating magnetic fields [2] [3].

Magnetic targeting refers to the attachment of therapeutic agents to magnetizable particles such as MNPs and then applying magnetic fields to concentrate them to the targeted region such as solid tumors and regions of infection [4]. The magnetic targeting of therapeutic agents results in the concentration of the therapy at the targeted site consequently reducing or eliminating the systemic drug side effects [5].

Accurate knowledge of the distribution and quantity of MNPs within the targeted tumor is crucial for effective and safe treatment planning of cancer therapy based on magnetic targeting [6]. Thus, the development of a readily available, noninvasive, and quantitative imaging technique will be necessary for the success of magnetic targeting. In 2005, a new imaging method called magnetic particle imaging (MPI) was introduced [7]. MPI allows imaging of the spatial distribution of MNPs, such as superparamagnetic iron oxide nanoparticles, with high sensitivity, spatial resolution, and imaging speed [7]. MPI uses the nonlinear response of MNPs to detect their presence in an alternating magnetic field, which is referred to here as the drive magnetic field. Spatial encoding is accomplished by saturating the MNPs over most of the imaged region using a static magnetic field (selection magnetic field), except in the vicinity of a special position called the field-free point [7] or field-free line [8]. We have developed a system for MPI with a field-free-line encoding scheme, in which the field-free line is generated using two opposing neodymium magnets, and transverse images are reconstructed from the third-harmonic signals received by a gradiometer coil using the maximum likelihood-expectation maximization (ML-EM) algorithm [9] [10].

The purpose of this study was to investigate the usefulness of MPI for monitoring the effect of magnetic targeting using tumor-bearing mice.

\section{Materials and Methods}

\subsection{System for Magnetic Particle Imaging}

The details of our MPI system are described in our previous papers [9] [10]. In brief, a drive magnetic field was generated using an excitation coil (solenoid coil $100 \mathrm{~mm}$ in length, $80 \mathrm{~mm}$ in inner diameter, and $110 \mathrm{~mm}$ in outer diameter). AC power was supplied to the excitation coil by a programmable power supply (EC1000S, NF CO., Yokohama, Japan), and was controlled using a sinusoidal wave generated by a digital function generator (DF1906, NF Co., Yokohama, Japan). The frequency and peak-to-peak strength of the drive magnetic field were taken as $400 \mathrm{~Hz}$ and $20 \mathrm{mT}$, respectively. The signal generated by MNPs was received by a gradiometer coil (50 $\mathrm{mm}$ in length, $35 \mathrm{~mm}$ in inner diameter, and $40 \mathrm{~mm}$ in outer diameter), and the third-harmonic signal was extracted using a preamplifier (T-AMP03HC, Turtle Industry Co., Ibaragi, Japan) and a lock-in amplifier (LI5640, NF Co., Yokohama, Japan). The output of the lock-in amplifier was converted to digital data by a personal computer connected to a multifunction data acquisition device with a universal serial bus port (USB-6212, National Instruments Co., TX, USA). The sampling time was taken as $10 \mathrm{~ms}$. When measuring signals using the gradiometer coil, a sample was placed $12.5 \mathrm{~mm}$ (i.e., a quarter of the coil length) from the center of the gradiometer coil and the coil, including the sample, was moved such that the center of the sample coincided with the position of the field-free line. The selection magnetic field was generated by two opposing neodymium magnets (Neomax Engineering Co., Gunma, Japan). The field-free line can be generated at the center of the two neodymium magnets.

To acquire projection data for image reconstruction, a sample (mouse) in the receiving coil was automatically rotated around the z-axis over $180^{\circ}$ in steps of $5^{\circ}$ and translated in the x-direction from $-16 \mathrm{~mm}$ to $16 \mathrm{~mm}$ in steps of $1 \mathrm{~mm}$, using an XYZ-axis rotary stage (HPS80-50X-M5, Sigma Koki Co., Tokyo, Japan), which was controlled using LabVIEW (National Instruments Co., TX, USA). Each projection data set was then transformed into 64 bins by linear interpolation. Both the inhomogeneous sensitivity of a receiving coil and feedthrough interference were corrected using the method described in [11]. Transverse images were reconstructed from the projection data using the ML-EM algorithm over 15 iterations, in which the initial concentration of MNPs was assumed to be uniform [9] [10]. 


\subsection{Magnetic Nanoparticles}

Resovist $^{\circledR}$ (FUJIFILM RI Pharma Co., Ltd., Tokyo, Japan) was used as the source of MNPs. Resovist ${ }^{\circledR}$ consists of MNPs (maghemite, $\gamma$ - $\mathrm{Fe}_{2} \mathrm{O}_{3}$ ) coated with carboxydextran [12]. It is an organ-specific contrast agent for magnetic resonance imaging (MRI), used especially for the detection of hepatocellular carcinoma and liver metastasis [12].

\subsection{Neodymium Magnet for Magnetic Targeting}

For magnetic targeting, a neodymium magnet (10 $\mathrm{mm}$ in diameter and $30 \mathrm{~mm}$ in length) (Sangyo Supply Co., Miyagi, Japan) was used.

Figure 1(a) shows the magnetic flux density perpendicular to the face of the neodymium magnet along the central axis. The closed circles and solid line represent the magnetic flux density measured using a Gauss meter (GM-301, Denshijiki Industry Co., Ltd., Tokyo, Japan) and that calculated using a software program based on the finite element method (COMSOL Multiphysics ${ }^{\circledR}$, COMSOL Inc., Stockholm, Sweden), respectively. The magnetic field strength on the surface of the neodymium magnet was $0.55 \mathrm{~T}$. Figure 1 (b) shows the contour plot of the magnetic flux density around the neodymium magnet, which was computed using COMSOL Multiphysics $^{\circledR}$ (COMSOL Inc., Stockholm, Sweden).

\subsection{Animal Experiments}

All animal experiments were approved by the animal ethics committee at Osaka University School of Medicine. Seven-week-old male BALB/c mice weighing $23.9 \pm 2.2 \mathrm{~g}$ (mean \pm standard deviation (SD)) were used. They were purchased from Charles River Laboratories Japan, Inc. (Yokohama, Japan), and were habituated to rearing environment for 1 week before the experiment. The animals had free access to food and water, and were kept under standard laboratory conditions of 22 - 23 degree room temperatures, around 50\% humidity, and a 12:12 hour light/dark cycle. Colon-26 cells $\left(1 \times 10^{6}\right.$ cells) were implanted subcutaneously into the mice under anesthesia by pentobarbital sodium (Somnopentyl, Kyoritsu Seiyaku Co., Tokyo, Japan) $(0.012 \mathrm{~mL} / \mathrm{g}$ body weight). Tumor volumes in all mice were measured by a caliper every day. The mice were divided into two groups and were used for

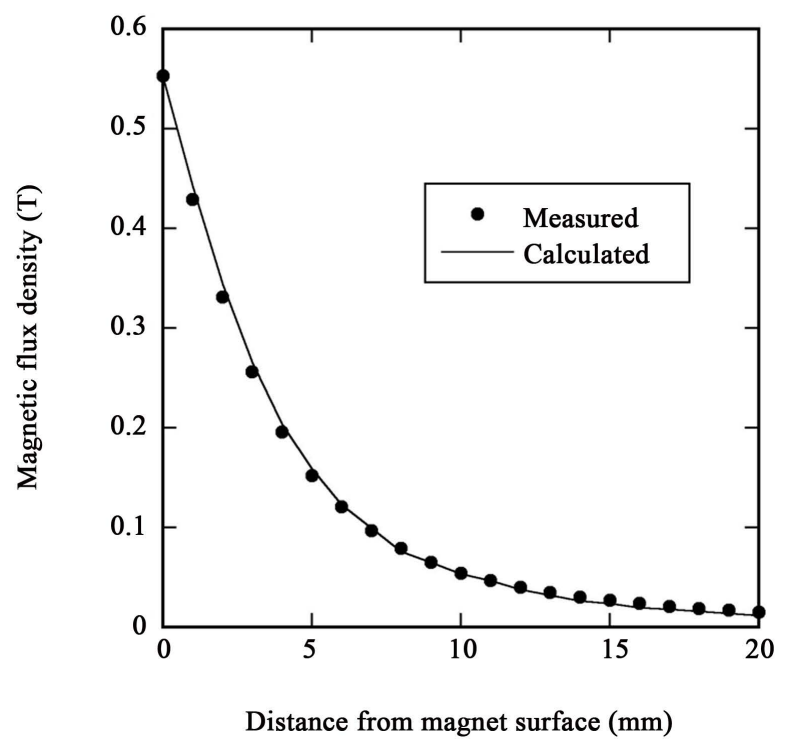

(a)

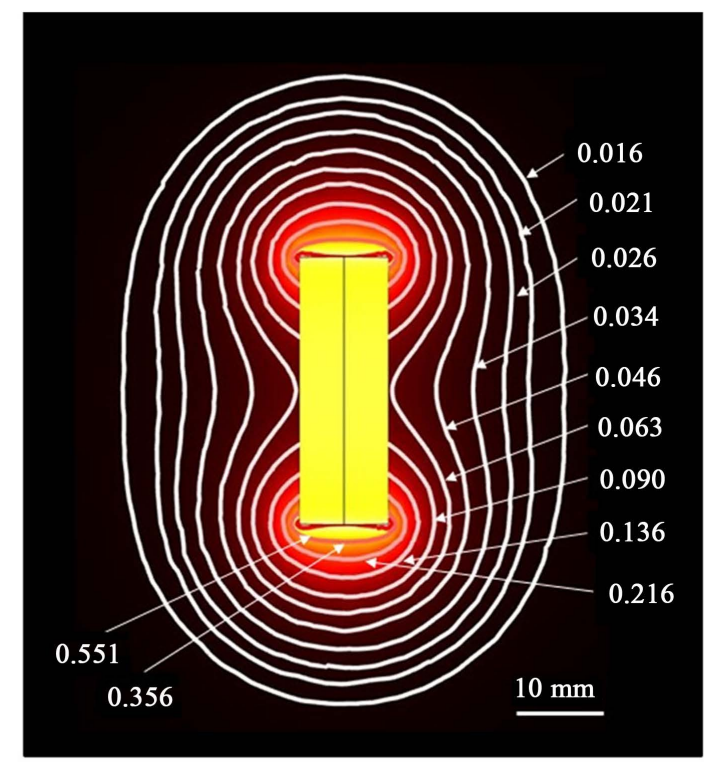

(b)

Figure 1. (a) Magnetic flux density perpendicular to the face of a neodymium magnet along the central axis. Closed circles and solid line represent the magnetic flux density measured using a Gauss meter and that calculated using a software program based on the finite element method (COMSOL Multiphysics ${ }^{\circledR}$, COMSOL Inc., Stockholm, Sweden), respectively; (b) Contour plot of the magnetic flux density around the neodymium magnet, computed by COMSOL Multiphysics ${ }^{\circledR}$. The numbers in the figure represent the magnetic flux density in Tesla. Scale bar $=10 \mathrm{~mm}$. 
experiments 7 to 10 days after implantation when the tumor volume reached approximately $100 \mathrm{~mm}^{3}$. The tumors in one group (treated group, $\mathrm{n}=8$ ) were directly injected with Resovist ${ }^{\circledR}$ with a concentration of $250 \mathrm{mM}(0.2 \mathrm{~mL})$ under anesthesia by pentobarbital sodium (Somnopentyl, Kyoritsu Seiyaku Co., Tokyo, Japan) (0.012 mL/g body weight) and the neodymium magnet (Figure 1(b)) was attached. The tumors in the other group (untreated group, $\mathrm{n}$ $=8$ ) were also directly injected with Resovist ${ }^{\circledR}$ in the same manner as in the treated group but the neodymium magnet was not attached. Figure 2 shows a photograph of our experimental setup for magnetic targeting. In the treated group, the neodymium magnet (Figure 1(b)) was attached to the tumor surface as shown in Figure 2.

Figure 3 shows the time schedule for data acquisition in this study. Each tumor-bearing mouse was scanned 5 times using our MPI scanner [9] [10]; 2 min, 37 min, 1 day, 3 days, and 7 days after the injection of Resovist ${ }^{\circledR}$ (Figure 3). After the MPI studies, X-ray CT images were obtained using a 4-row multi-slice CT scanner (Asteion, Toshiba Medical Systems Co., Tochigi, Japan) with a tube voltage of $120 \mathrm{kV}$, a tube current of $210 \mathrm{~mA}$, and a slice thickness of $0.5 \mathrm{~mm}$. The MPI image was co-registered with the X-ray CT image using the parameters for magnification and rotation, which were obtained using a phantom with 3 point sources with a diameter of $0.5 \mathrm{~mm}$ and filled with $100 \mathrm{mM}$ MNPs [13]. It should be noted that the neodymium magnet (Figure 1(b)) was attached to the tumor surface for 20 min per event in the treated group and that the X-ray CT image after the first MPI study was substituted by that obtained after the second MPI study.

As previously described, tumor volumes in all mice were measured by a caliper every day. The tumor volume $\left(V\right.$ in $\mathrm{mm}^{3}$ ) was calculated as $V=(\pi / 6) \times L_{\mathrm{x}} \times L_{\mathrm{y}} \times L_{\mathrm{z}}$, where $L_{\mathrm{x}}, L_{\mathrm{y}}$, and $L_{\mathrm{z}}$ denote the vertical diameter, the horizontal diameter, and the height in mm, respectively. The relative tumor volume growth (RTVG) was calculated from $\left(V-V_{0}\right) / V_{0}$, where $V_{0}$ represents the tumor volume immediately before the injection of Resovist ${ }^{\circledR}$.

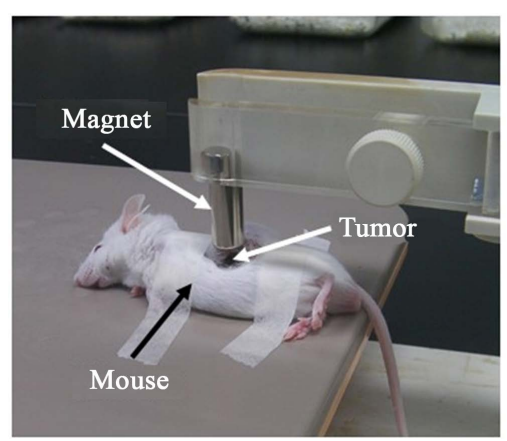

Figure 2. Photograph of our experimental setup for magnetic targeting.

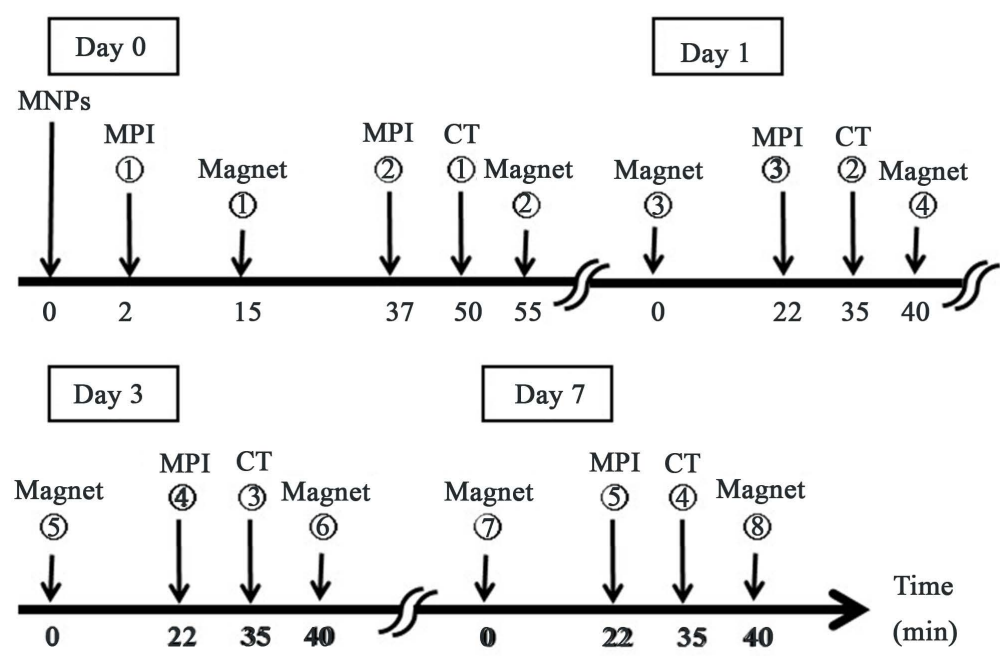

Figure 3. Time schedule for data acquisition. MNPs: magnetic nanoparticles, MPI: magnetic particle imaging, Magnet: attachment of a neodymium magnet to the tumor surface, and CT: X-ray computed tomography. In the treated group, the neodymium magnet was attached to the tumor surface for $20 \mathrm{~min}$ per event, whereas the magnet was not attached to the tumor in the untreated group. 


\subsection{Data and Statistical Analyses}

After the MPI studies, we drew a region of interest (ROI) on the tumor in the MPI image and calculated the average and maximum MPI values within the ROI by taking the threshold value for extracting the contour of the tumor as $40 \%$ of the maximum MPI value in the ROI. The average MPI value was calculated by dividing the total pixel values by the number of pixels within the ROI. In this study, the MPI value was defined as the pixel value of the transverse image reconstructed from the third-harmonic signals. The average MPI value had an excellent linear correlation with the iron concentration of Resovist ${ }^{\circledR}$ [13].

The RTVG, average MPI value, and maximum MPI value were expressed as mean \pm standard error (SE). Differences in these parameters between the untreated and treated groups were analyzed by unpaired Student's $t$-test. A $p$-value less than 0.05 was considered statistically significant. When a $p$-value was greater than 0.05 and less than 0.1 , it was considered marginally significant.

\section{Results}

Figure 4 shows typical examples of the MPI images of tumor-bearing mice injected with $250 \mathrm{mM}$ Resovist ${ }^{\circledR} 2$ min, 37 min, 1 day, 3 days, and 7 days after the injection of Resovist ${ }^{\circledR}$, which were superimposed on the X-ray CT images. The upper and lower panels show the images in the untreated and treated groups, respectively. As shown in Figure 4, the pixel values in the MPI images decreased with time in both groups. When the neodymium magnet was attached to the tumor surface (lower panels), the clearance of Resovist ${ }^{\circledR}$ in the tumor was somewhat reduced as compared to the case when the magnet was not attached (upper panels).

Figure 5 shows the time courses of the RTVG value in the untreated (closed circles) and treated groups (open circles). There were no significant differences between the two groups throughout the study period.

Figure 6 shows the comparison of the average MPI values between the untreated and treated groups 2 min, 37 min, 1 day, 3 days, and 7 days after the injection of Resovist ${ }^{\circledR}$. It should be noted that the average MPI value 2 min after the injection of Resovist ${ }^{\circledR}$ was normalized as unity. The average MPI value in the treated group was significantly higher $(p=0.044)$ than that in the untreated group 3 days after the injection of Resovist ${ }^{\circledR}$. There was a marginally significant difference $(p=0.089)$ between the two groups 1 day after the injection of Resovist ${ }^{\circledR}$.

Figure 7 shows the comparison of the maximum MPI values between two groups 2 min, 37 min, 1 day, 3 days, and 7 days after the injection of Resovist ${ }^{\circledR}$. As in Figure 6, the maximum MPI value 2 min after the injection of Resovist ${ }^{\circledR}$ was normalized as unity. The maximum MPI value in the treated group was significantly higher ( $p=0.037$ ) than that in the untreated group 3 days after the injection of Resovist ${ }^{\circledR}$. There was a marginally significant difference ( $p=0.076$ ) between the two groups 1 day after the injection of Resovist ${ }^{\circledR}$.
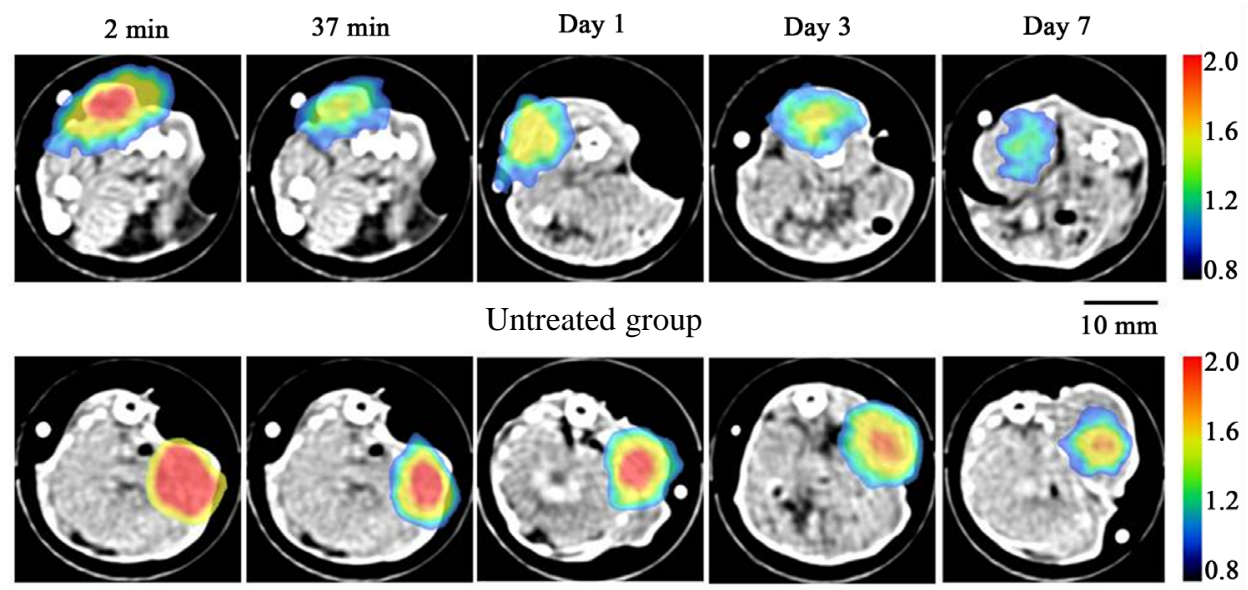

Untreated group

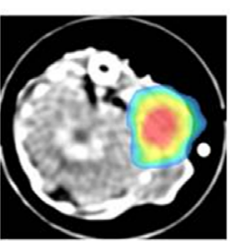

Treated group
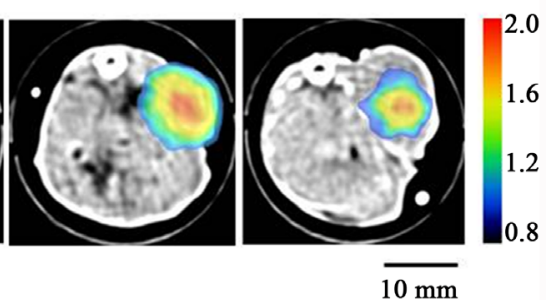

Figure 4. Typical examples of the MPI images superimposed on the X-ray CT images in the untreated (upper panels) and treated groups (lower panels) $2 \mathrm{~min}, 37 \mathrm{~min}, 1$ day, 3 days, and 7 days after the injection of Resovist ${ }^{\circledR}$. The mice in the treated group were intratumorally injected with $250 \mathrm{mM}$ Resovist ${ }^{\circledR}(0.2 \mathrm{~mL})$ and a neodymium magnet was attached to the tumor surface for 20 min per event (refer to Figure 3 ), whereas the magnet was not attached to the tumor surface in the untreated group. 


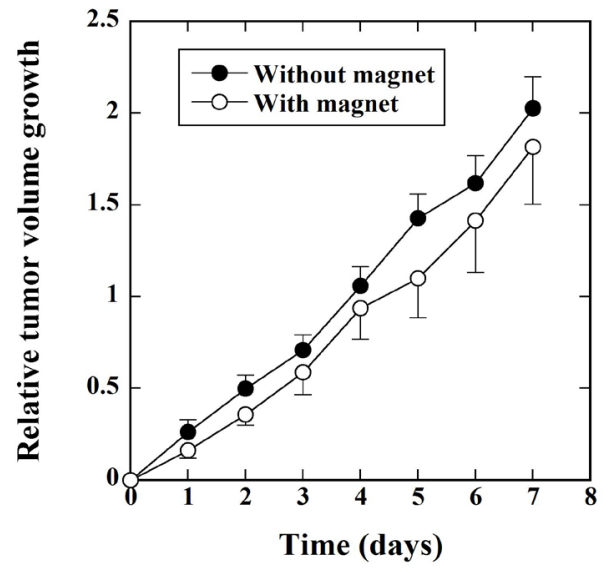

Figure 5. Time courses of the relative tumor volume growth (RTVG) in the untreated (closed circles) and treated groups (open circles). The RTVG was calculated from $\left(V-V_{0}\right) / V_{0}$, where $V_{0}$ and $V$ represent the tumor volume immediately before and after the injection of Resovist ${ }^{\circledR}$, respectively. Data are represented by mean \pm standard error $(\mathrm{SE})$ for $\mathrm{n}=8$.

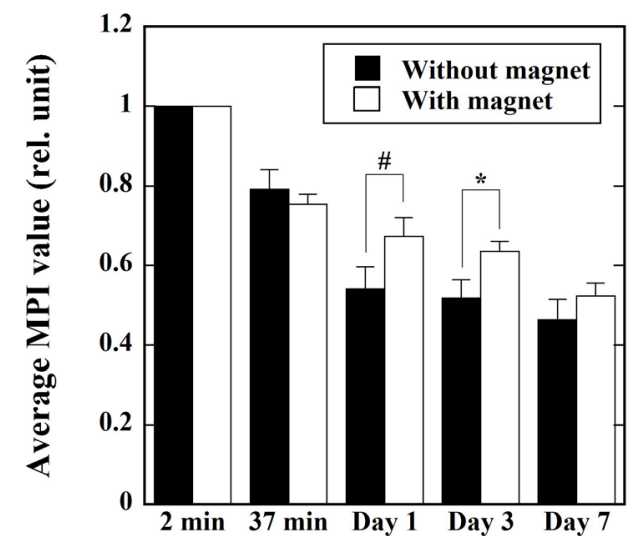

Figure 6. Comparison of the average MPI values between the untreated (closed bars) and treated groups (open bars) 2 min, 37 min, 1 day, 3 days, and 7 days after the injection of Resovist ${ }^{\circledR}$. Bar and error bar represent mean and $\mathrm{SE}$ for $\mathrm{n}=8$, respectively. Note that the average MPI value 2 min after the injection of Resovist ${ }^{\circledR}$ was normalized as unity. ${ }^{*} p<0.05$ and ${ }^{\#} p=0.089$.

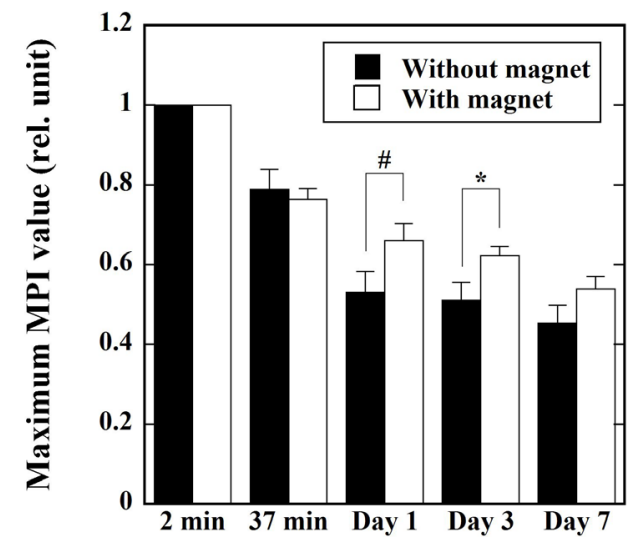

Figure 7. Comparison of the maximum MPI values between the untreated (closed bars) and treated groups (open bars) 2 min, 37 min, 1 day, 3 days, and 7 days after the injection of Resovist ${ }^{\circledR}$. Bar and error bar represent mean and SE for $n=8$, respectively. Note that the maximum MPI value 2 min after the injection of Resovist ${ }^{\circledR}$ was normalized as unity. ${ }^{*} p<0.05$ and ${ }^{\#} p=0.076$. 


\section{Discussion}

In this study, we investigated the usefulness of MPI for monitoring the effect of magnetic targeting using tumorbearing mice. Our preliminary results (Figure 6 and Figure 7) demonstrated that when a neodymium magnet was attached to the tumor surface, the average and maximum MPI values in the tumor were significantly higher than those when the magnet was not attached, 3 days after the injection of Resovist ${ }^{\circledR}$. These results suggest that MPI is applicable and useful for monitoring the effect of magnetic targeting. To the best of our knowledge, this is the first report on the application of MPI to monitoring the effect of magnetic targeting.

We previously reported that there is an excellent linear correlation between the average MPI value and the iron concentration of Resovist ${ }^{\circledR}$ in phantom studies [13]. From this finding, it appears that the change in the average MPI value corresponds to that in the amount of MNPs per voxel in the selected slice of the tumor. Thus, the results shown in Figure 6 and Figure 7 suggest that when a magnet was attached to the tumor surface, a greater amount of MNPs was retained in the tumor than the case when the magnet was not attached.

As shown in Figure 5, there were no significant differences in the RTVG value between the untreated and treated groups. Thus, it appears that the above findings shown in Figure 6 and Figure 7 are not related to the tumor volume growth.

The success of MNP-based cancer therapy depends on the ability to deliver MNPs systematically to tumor cells in sufficient concentrations. This is due in part to the presence of the inherent leakiness of the tumor vasculature, the so-called enhanced permeability and retention (EPR) effect. Although the EPR effect is present in varying degrees in many tumors, it has not resulted in the consistent level of MNP tumor uptake [14]. Thus, the delivery of adequate numbers of MNPs to the tumor site via systemic administration remains challenging. If MNPs were adsorbed only to tumor cells, the MNPs could be administered intravenously. This feature would be of great advantage in terms of the quality of life of patients. However, because the administered MNPs migrate passively to a mononuclear phagocyte system such as the Kupffer cells of the liver and spleen, the passive targeting of MNPs for cancer is a very important issue for the establishment of MNP-based cancer therapy. With regard to the delivery of MNPs, magnetoliposomes may be a promising tool for passive targeting. Shinkai et al. [15] developed magnetite cationic liposomes (MCLs) with improved absorption and accumulation properties within tumors. Administration of the MCLs, however, is limited to direct injection into the tumor tissue [16]. The conjugation of antibodies to MNPs is a possible approach to achieving the passive targeting of MNPs for cancer. Le et al. [17] and Shinkai et al. [18] have developed MNPs conjugated to the Fab' fragments of anti-human MN antigen-specific antibody. However, because the target concentration is very low in antibody targeting of tumors [19], this approach cannot yet be translated successfully from research to the clinical stage. If we combined these approaches and magnetic targeting, it might be able to contribute to the success of MNPbased cancer therapy.

Ionizing radiation, anticancer drugs such as cisplatin, and vascular disrupting agents are being used to modify the tumor environment/vasculature barrier to improve MNP uptake in tumors and subsequently tumor treatment [14]. Recently, we investigated the usefulness of the combination of ionizing radiation or vascular disrupting agent and magnetic hyperthermia treatment (MHT) using tumor-bearing mice, and reported that these methods are useful not only for improving the therapeutic effect of MHT but also for enhancing the retention of MNPs in the tumor [20] [21], which will be useful especially for repeated applications of MHT. Magnetic targeting with use of an external static magnetic field as done in this study, however, appears to be easier to implement and more advantageous in terms of safety than the use of ionizing radiation or vascular disrupting agent.

The limitation of this study is that the average and maximum MPI values were obtained from a single slice with the maximum signal intensity. For more detailed analysis, three-dimensional (multi-slice) data will be necessary. If these three-dimensional data are obtained, the accuracy of MPI for monitoring the effect of magnetic targeting will be further enhanced. These studies are now in progress. In addition, we injected Resovist ${ }^{\circledR}$ directly into the tumor in this study, because an intravenous-injection approach to achieving the passive targeting of MNPs for cancer is not yet available in the clinical setting, as previously described. Thus, the present results are limited to the case when the MNPs were injected directly into the tumor. We are also currently studying the case when the MNPs are injected intravenously.

Another imaging method to detect MNPs is magnetic resonance imaging (MRI). Some investigators attempted to use MRI for tracking the location of magnetically labeled cells [22] [23]. They also attempted to use the magnetic field gradient coils inherent to all MRI systems, for steering MNPs to a targeted site [22] [23]. 
When we attempted to image MNPs using MRI with a conventional transverse relaxation time $\left(T_{2}^{*}\right)$-weighted imaging sequence, it was almost impossible due to large susceptibility-induced MR signal loss and image distortions in the regions near the MNPs. Recently, however, MRI pulse sequences capable of preserving signal from spins with ultrashort $T_{2}{ }^{*}$, such as ultrashort echo time (UTE) [24] and sweep imaging with Fourier transformation (SWIFT) sequences [25], have been developed. With these pulse sequences, MNPs can be detected and quantified based on the shortening of the longitudinal relaxation time of water $\left(T_{1}\right)$ [25]. Zhang et al. [26] reported that the reciprocal of $T_{1}$ measured using the SWIFT sequence combined with the Look-Locker method has a linear relationship with MNPs concentration up to $53.6 \mathrm{mM}$ of iron. Although their method also appears to be promising and useful for monitoring the effect of magnetic targeting, significant research and technology development remain to be done for establishing the effectiveness of their method when translated to animal and/or human studies [14].

\section{Conclusion}

Our preliminary results suggest that MPI can be useful for monitoring the effect of magnetic targeting and will provide a new imaging strategy for establishing MNP-based cancer therapy as well as MNP-based drug delivery using magnetic targeting in the clinical setting.

\section{Acknowledgements}

This work was supported by a Grant-in-Aid for Scientific Research (Grant Number: 25282131 and 15K12508) from the Japan Society for the Promotion of Science (JSPS) and Japan Science and Technology Agency (JST).

\section{Declaration of Interest}

The authors report no conflicts of interest.

\section{References}

[1] Vauthier, C., Labarre, D. and Ponchel, G. (2007) Design Aspects of Poly(alkylcyanoacrylate) Nanoparticles for Drug Delivery. Journal of Drug Targeting, 15, 641-663. http://dx.doi.org/10.1080/10611860701603372

[2] Kong, S.D., Lee, J., Ramachandran, S., Eliceiri, B.P., Shubayev, V.I., Lal, R. and Jin, S. (2012) Magnetic Targeting of Nanoparticles across the Intact Blood-Brain Barrier. Journal of Controlled Release, 164, 49-57. http://dx.doi.org/10.1016/j.jconrel.2012.09.021

[3] Jordan, A., Scholz, R., Maier-Hauff, K., Johannsen, M., Wust, P., Nodobny, J., Schirra, H., Schmidt, H., Deger, S., Loening, S., Lanksch, W. and Felix, R. (2001) Presentation of a New Magnetic Field Therapy System for the Treatment of Human Solid Tumors with Magnetic Fluid Hyperthermia. Journal of Magnetism and Magnetic Materials, 225, 118-126. http://dx.doi.org/10.1016/S0304-8853(00)01239-7

[4] Nacev, A., Beni, C., Bruno, O. and Shapiro, B. (2011) The Behaviors of Ferro-Magnetic Nano-Particles in and around Blood Vessels under Applied Magnetic Fields. Journal of Magnetism and Magnetic Materials, 323, 651-668. http://dx.doi.org/10.1016/j.jmmm.2010.09.008

[5] Polyak, B. and Friedman, G. (2009) Magnetic Targeting for Site-Specific Drug Delivery: Applications and Clinical Potential. Expert Opinion on Drug Delivery, 6, 53-70. http://dx.doi.org/10.1517/17425240802662795

[6] Johannsen, M., Gneueckow, U., Thiesen, B., Taymoorian, K., Cho, C.H., Waldofner, N., Scholz, R., Jordan, A., Loening, S.A. and Wust, P. (2007) Thermotherapy of Prostate Cancer Using Magnetic Nanoparticles: Feasibility, Imaging, and Three-Dimensional Temperature Distribution. European Urology, 52, 1653-1662. http://dx.doi.org/10.1016/j.eururo.2006.11.023

[7] Gleich, B. and Weizenecker, J. (2005) Tomographic Imaging Using the Nonlinear Response of Magnetic Particles. Nature, 435, 1214-1217. http://dx.doi.org/10.1038/nature03808

[8] Goodwill, P.W., Konkle, J.J., Zheng, B., Saritas, E.U. and Conolly, S.M. (2012) Projection X-Space Magnetic Particle Imaging. IEEE Transactions on Medical Imaging, 31, 1076-1085. http://dx.doi.org/10.1109/TMI.2012.2185247

[9] Murase, K., Hiratsuka, S., Song R. and Takeuchi, Y. (2014) Development of a System for Magnetic Particle Imaging Using Neodymium Magnets and Gradiometer. Japanese Journal of Applied Physics, 53, Article ID: 067001. http://dx.doi.org/10.7567/JJAP.53.067001

[10] Murase, K., Song, R. and Hiratsuka, S. (2014) Magnetic Particle Imaging of Blood Coagulation. Applied Physics Letters, 104, Article ID: 252409. http://dx.doi.org/10.1063/1.4885146 
[11] Murase, K., Banura, N., Mimura, A. and Nishimoto, K. (2015) Simple and Practical Method for Correcting the Inhomogeneous Sensitivity of a Receiving Coil in Magnetic Particle Imaging. Japanese Journal of Applied Physics, 54, Article ID: 038001. http://dx.doi.org/10.7567/JJAP.54.038001

[12] Murase, K., Oonoki, J., Takata, H., Song, R., Angraini, A., Ausanai, P. and Matsushita, T. (2011) Simulation and Experimental Studies on Magnetic Hyperthermia with Use of Superparamagnetic Iron Oxide Nanoparticles. Radiological Physics and Technology, 4, 194-202. http://dx.doi.org/10.1007/s12194-011-0123-4

[13] Murase, K., Aoki, M., Banura, N., Nishimoto, K., Mimura, A., Kuboyabu, T. and Yabata, I. (2015) Usefulness of Magnetic Particle Imaging for Predicting the Therapeutic Effect of Magnetic Hyperthermia. Open Journal of Medical Imaging, 5, 85-99. http://dx.doi.org/10.4236/ojmi.2015.52013

[14] Hoopes, P.J., Petryk, A.A., Tate, J.A., Savellano, M.S., Strawbridge, R.R., Giustini, A.J., Stan, R.V., Gimi, B. and Garwood, M. (2013) Imaging and Modification of the Tumor Vascular Barrier for Improvement in Magnetic Nanoparticle Uptake and Hyperthermia Treatment Efficacy. Proceedings SPIE Energy-Based Treatment of Tissue and Assessment VII, 8584, Article ID: 858403. http://dx.doi.org/10.1117/12.2008689

[15] Shinkai, M., Yanase, M., Honda, H., Wakabayashi, T., Yoshida, J. and Kobayashi, T. (1996) Intracellular Hyperthermia for Cancer Using Magnetite Cationic Liposome: in Vitro Study. Japanese Journal of Cancer Research, 87, 11791183. http://dx.doi.org/10.1111/j.1349-7006.1996.tb03129.x

[16] Ito, A., Shinkai, M., Honda, H. and Kobayashi, T. (2005) Medical Applications of Functionalized Magnetic Nanoparticles. Journal of Bioscience and Bioengineering, 100, 1-11. http://dx.doi.org/10.1263/jbb.100.1

[17] Le, B., Shinkai, M., Kitade, T., Honda, H., Yoshida, J., Wakabayashi, T. and Kobayashi, T. (2001) Preparation of Tumor-Specific Magnetoliposomes and Their Application for Hyperthermia. Journal of Chemical Engineering of Japan, 34, 66-72. http://dx.doi.org/10.1252/jcej.34.66

[18] Shinkai, M., Le, B., Honda, H., Yoshikawa, K., Shimizu, K., Saga, S., Wakabayashi, T., Yoshida, J. and Kobayashi, T. (2001) Targeting Hyperthermia for Renal Cell Carcinoma Using Human MN Antigen-Specific Magnetoliposomes. Japanese Journal of Cancer Research, 92, 1138-1146. http://dx.doi.org/10.1111/j.1349-7006.2001.tb01070.x

[19] Suzuki, M., Shinkai, M., Kamihira, M. and Kobayashi, T. (1995) Preparation and Characteristics of Magnetite-Labelled Antibody with the Use of Poly (Ethylene Glycol) Derivatives. Biotechnology and Applied Biochemistry, 21, 335-345.

[20] Ohki, A., Kuboyabu, T., Yamawaki, M., Aoki, M. and Murase, K. (2016) Quantitative Evaluation of Tumor Response to Combination of Magnetic Hyperthermia Treatment and Radiation Therapy Using Magnetic Particle Imaging. 12th International Congress of Hyperthermic Oncology (ICHO2016), New Orleans, 11-15 April 2016, Abstract Book, p. 166.

[21] Kuboyabu, T., Yamawaki, M., Ohki, A., Aoki, M. and Murase, K. (2016) Quantitative Evaluation of Tumor Early Response to Magnetic Hyperthermia Treatment Combined with Vascular Disrupting Therapy Using a Newly Developed Method for Magnetic Particle Imaging. 12th International Congress of Hyperthermic Oncology (ICHO2016), New Orleans, 11-15 April 2016, Abstract Book, p. 167.

[22] Riegler, J., Wells, J.A., Kyrtatos, P.G., Price, A.N., Pankhurst, Q.A. and Lythgoe, M.F. (2010) Targeted Magnetic Delivery and Tracking of Cells Using a Magnetic Resonance Imaging System. Biomaterials, 31, 5366-5371. http://dx.doi.org/10.1016/j.biomaterials.2010.03.032

[23] Muthana, M., Kennerley, A.J., Hughes, R., Fagnano, E., Richardson, J., Paul, M., Murdoch, C., Wright, F., Payne, C., Lythgoe, M.F., Farrow, N., Dobson, J., Conner, J., Wild, J.M. and Lewis, C. (2015) Directing Cell Therapy to Anatomic Target Sites in Vivo with Magnetic Resonance Targeting. Nature Communications, 6, Article Number: 8009. http://dx.doi.org/10.1038/ncomms9009

[24] Robson, M.D., Gatehouse, P.D., Bydder, M. and Bydder, G.M. (2003) Magnetic Resonance: An Introduction to Ultrashort TE (UTE) Imaging. Journal of Computer Assisted Tomography, 27, 825-846. http://dx.doi.org/10.1097/00004728-200311000-00001

[25] Idiyatullin, D., Corum, C., Park, J.Y. and Garwood, M. (2006) Fast and Quiet MRI Using a Swept Radiofrequency. Journal of Magnetic Resonance, 181, 342-349. http://dx.doi.org/10.1016/j.jmr.2006.05.014

[26] Zhang, J., Chamberlain, R., Etheridge, M., Idiyatullin, D., Corum, C., Bischof, J. and Garwood, M. (2014) Quantifying Iron-Oxide Nanoparticles at High Concentration Based on Longitudinal Relaxation Using a Three-Dimensional SWIFT Look-Locker Sequence. Magnetic Resonance in Medicine, 71, 1982-1988. http://dx.doi.org/10.1002/mrm.25181 